\title{
Littérature et anthropologie
}

\section{Avant-propos}

Silvia Disegni

\section{OpenEdition}

Journals

Édition électronique

URL : http://journals.openedition.org/recherchestravaux/563

DOI : 10.4000/recherchestravaux.563

ISSN : 1969-6434

\section{Éditeur}

UGA Éditions/Université Grenoble Alpes

\section{Édition imprimée}

Date de publication : 15 mai 2013

Pagination : 5-10

ISBN : 978-2-84310-248-6

ISSN : 0151-1874

\section{Référence électronique}

Silvia Disegni, «Avant-propos », Recherches \& Travaux [En ligne], 82 | 2013, mis en ligne le 15 novembre 2014, consulté le 25 septembre 2020. URL : http://journals.openedition.org/recherchestravaux/563 ;

DOI : https://doi.org/10.4000/recherchestravaux.563 
Silvia Disegni

Université Federico II, Naples

\section{Avant-propos}

Nous réunissons ici les actes d'une rencontre organisée à Naples à l'université Federico II les 4 et 5 mars 20IO, intitulée Letteratura e antropologia (Italia-Francia), réalisée en collaboration avec l'Institut français de Naples, le Laboratoire d'anthropologie et d'histoire de l'institution de la culture (LAHIC), l'université Paul Verlaine de Metz et l'Istituto di Studi filosofici de Naples. Il s'agissait pour nous d'établir un triple dialogue entre les chercheurs réunis : en premier lieu entre littéraires et anthropologues à un moment où se multiplient les questionnements sur les rapports entre littérature et anthropologie ${ }^{\mathrm{r}}$ qui succèdent à ceux que l'on avait tenté d'établir ces dernières années entre la littérature et d'autres sciences humaines et sociales comme la psychologie ou la psychanalyse, la sociologie, l'histoire, par exemple; d'autre part, entre certains représentants des deux disciplines appartenant à deux espaces géographiques et culturels différents, l'Italie et la France où généralement et malgré l'ère de la globalisation, n’ont pas été choisis les mêmes approches, démarches ou instruments, les mêmes références ${ }^{2}$ (en Italie Gramsci, De Martino, Cirese

I. Pour ne citer que quelques récents numéros spéciaux de revue : L'Homme, nº 175 -176, Vérités de la fiction, 2005; Romantisme, n' I4I, Ethnocritique de la littérature, 2009; Pratiques, $\mathrm{n}^{\circ}$ I5I-I52, Anthropologies de la littérature, décembre 20II. Et encore les collectifs : Littérature et anthropologie, A. Montandon (dir.), Paris, Champ social éd., 2006; Horizons ethnocritiques, J.-M. Privat, M. Scarpa, Presses universitaires de Nancy, 20I0; Savoirs romantiques. Une naissance de l'anthropologie, D. Fabre, J.-M. Privat (dir.), Nancy, Presses universitaires de Nancy, 20II ; voir aussi, dans le dernier numéro de L'Homme ( ${ }^{\circ}$ 203-204, 20I2, 3-4), l'article de D. Fabre et J. Jamin : "Quelques considérations sur les rapports entre anthropologie et littérature", p. 579-6I2 (sans oublier le moins récent numéro spécial de L'Homme, no III-II2, Littérature et anthropologie, 1989).

2. Des renseignements sur l'anthropologie italienne sont fournis dans P. Clemente, «Les anthropologues italiens et l'Italie», Terrains, $\mathrm{n}^{\circ}$ I2, 1989. Quelques titres sur notre sujet : D. Scafoglio, Antropologia e letteratura, I, II, III, Salerne, Gentile, 1996, 2000, 2002 ; autour 
surtout et l'anthropologie anglo-américaine en particulier pour le rapport que nous envisageons ici; en France, Mauss, Lévi-Strauss et le débat avec l'anthropologie anglo-américaine, par exemple). D'où sans doute, dans le passé, «les rendez-vous manqués» entre les tenants de la même discipline des deux pays, pour employer l'expression de Daniel Fabre 3 , ou des lectures plus tardives de certains textes qui avaient joué un rôle dans leur propre pays au moment de leur publication; enfin, entre tenants de la même discipline mais dont l'objet de spécialité ne se situe pas au même moment de l'histoire, les documents ne sont pas de même nature, les visions du monde ne sont pas superposables ni sans doute le rapport entre les deux disciplines à différents moments de l'histoire, malgré une approche thématique partagée par certains. Le projet pouvait paraître ambitieux et donner l'idée d'une «expérience à instituer ${ }^{4}$ » comme l'écrit Zola ou encore d'une situation typiquement anthropologique où l'on est à l'écoute de «l'autre» que soi, ou plutôt d'autres que soi (étant donné les différentes typologies d' «autres» en présence), quoique "proches» (étant donné le voisinage des deux cultures, des deux domaines étudiés). Évidemment, ni la courte durée de ces rencontres ni le nombre réduit des chercheurs qui ne pouvaient couvrir les différents cas de figure envisagés ne pouvaient permettre de réaliser pleinement ce projet fantasmatique (l'auraient-ils pu, d'ailleurs?). Ce numéro voudrait apporter le témoignage de la vivacité et de la qualité des débats, tout comme de la variété des approches.

L'objet commun a été trouvé, tant dans les textes plus théoriques que dans les analyses de cas : le texte littéraire, qui s'est à vrai dire imposé sans même avoir été convoqué, et surtout, on pourrait s’en étonner, le texte légitimé (au sens de Pierre Bourdieu) : le récit ou roman surtout (Champfleury, Flaubert,

de l'importante réception de Frazer en Italie : F. Dei, le chapitre «Antropologia e letteratura" dans La discesa agli inferi: James Frazer e la cultura del novecento, Lecce, Argo, 1998 (disponible depuis 2009 sur <www.fareantropologia.it>) ; R. Gambino, Antropologia letteraria, dans <www. culturalstudies.it>; A. M. Sobrero, Il cristallo e la fiamma : antropologia fra scienza e letteratura, Rome, Carocci 2009 ; G. Burali, Labili confini tra scrittura: antropologia e letteratura disponible sur <www.solima.media.unisi.it> [consulté le 28/03/2013] ; voir aussi une bibliographie utile : Letteratura italiana e antropologia : percorsi bibliografici disponible sur <www.thefreelibrary. com> [consulté le 28/03/20I3]; rappelons l'importance de Gramsci en Italie dans le domaine anthropologique pour sa réflexion sur la culture populaire (voir ses Cahiers de prison, Paris, Gallimard, 5 vol., 1978-1996); nous renvoyons évidemment aux études fondamentales menées par l'anthropologue A. M. Cirese sur la littérature populaire à partir de 1954 jusqu'à sa mort en 200I. Voir aussi les ouvrages d'E. De Martino dont La fine del mondo. Contributo all'analisi delle apocalissi culturali [1977], Turin, Einaudi, 2002 pour l'importance accordée aux textes littéraires (et en particulier français) dans l'analyse anthropologique du thème de la fin du monde.

3. D. Fabre, «Un rendez-vous manqué. Francesco de Martino et sa réception en France», L'Homme, $\mathrm{n}^{\circ}$ I5I-152, 1999.

4. É. Zola, Le Roman expérimental [I88I], Paris, Garnier Flammarion, 2006, p. 56. 
Goncourt, Zola, Wiesel, Le Bon et, dans le domaine italien, Verga, Tomasi di Lampedusa); l'autobiographie (Leiris); l'un des deux textes qu'écrivent les ethnologues français au retour de leur mission (Lévi-Strauss); mais aussi, dans l'étude thématique et panoramique de Carlo Donà, des récits de voyages par exemple. Or on sait que le texte littéraire est au cœur de la formation, non seulement des chercheurs en littérature mais aussi de la plupart des anthropologues et à plus forte raison des écrivains anthropologues dont le nombre a considérablement augmenté ces derniers temps.

On a souvent constaté qu'il y a eu, au début du $\mathrm{xx}^{\mathrm{e}}$ siècle, conflit entre les deux domaines, surtout de la part des anthropologues soucieux de revendiquer leur statut scientifique : la littérature, conçue comme produit de l'imagination, comme fiction, subjective, devient alors ce dont il faut se distinguer au nom du fait réel et de la vérité; conçue comme un art pourvu de règles, comme le produit d'une rhétorique, elle masque les faits derrière ses dentelles, ses figures, ses recherches d'effet qui compromettent toute objectivité. Mais un tel discours, au fond, n'est pas sans faire penser, au sein même du champ littéraire, aux déclarations de principe des tenants du réalisme et surtout du naturalisme, désireux de s'affranchir du romantisme, trop lié à l'imagination subjective, aux effets grossissants et ne pouvant mettre l'excès de son esthétique au service de la "vérité observable». On sait d'ailleurs que les naturalistes ont été apparentés à l'anthropologie par leur démarche (l'enquête nécessaire à la constitution du roman, la recherche du "document humain", expression à laquelle les anthropologues ont pu souscrire); leurs méthodes (l'observation, la prise de notes, le passage à l'écriture, au texte où se donne à lire une distance énonciative en même temps qu'une profusion de détails, de renseignements organisés en système); leurs objets (les "primitifs» de proximité) et qui, comme les anthropologues, entend en porter à la connaissance de l'homme. On comprend alors que la littérature réaliste ait pu servir de réservoir de documents considérés comme dignes d'analyse par certains anthropologues, parfois même plus souvent que des textes romantiques, pourtant plus attachés au folklore mais moins fiables car filtrés par une subjectivité plus exubérante et moins soumis aux contraintes de "la science». Mais de même qu'on est vite revenu de la scientificité du réalisme et du naturalisme dans le domaine littéraire, car l'écrivain accumule dans ses romans mythes et fantasmes et soumet les données de ses enquêtes à une sémantisation et à une esthétisation d'autant plus fortes qu'elles sont moins visibles, de même on est revenu d'une conception de l'anthropologie d'où la subjectivité serait exclue, pour en arriver à une situation complètement inversée, comme le souligne Daniel Fabre qui parle alors d'indistinction entre les deux domaines. C'est, en effet, ce qui se donne à lire dans les textes de toute une tendance de l'anthropologie américaine, mais aussi dans des romans ou 
autobiographies d'ethnologues, voire des romans où l'anthropologie n'est plus convoquée, comme le montre Gilles Bonnet, pour la description de rites obsolètes et rassurants face à la mort, mais parce qu'elle contribue à la révélation de l'Autre, du mort qui est en soi, dans un contexte de déracinement et dans la plus grande intériorité.

Ce volume est composé de quatre parties.

Dans la première, sont réunis des textes aux visées plus théoriques. Daniel Fabre part de l'analyse du Guépard, conçu comme roman de "l'ultime», pour développer une catégorie empruntée à l'anthropologie, science qui «n'est pas tant la discipline de l'altérité géographique que celle des ruptures séculaires dont témoignent sa propre naissance et une pratique qui s'est lentement mondialisée». Marie Scarpa expose les principes de l'ethnocritique, que Jean-Marie Privat utilisera plus loin dans une analyse de la "casquette» de Madame Bovary : dire, comme elle le fait, que «l'ethnocritique se préoccupe de ce que "la littérature fait à l'anthropologie" présuppose une attention particulière portée au poiein de l'écrivain, attention qui fait de l'anthropologie l'un des éléments de la création romanesque : «nous faisons l'hypothèse que les traits de culture présents dans l'œuvre littéraire [...] s'organisent en systèmes discursifs et en cosmologies culturelles, toujours métissés et pluriels. Notre objet est donc l'analyse de cette dialogisation, au sein de l'œuvre, d'univers symboliques plus ou moins hétérogènes et hybrides». Romano Luperini s'interroge quant à lui sur les derniers avatars de l'analyse thématique, qu'il aborde à trois niveaux : "la notion de thème oscille entre deux pôles : "l'argument" d'un côté, et "l'idée inspiratrice" de l'autre»; "entre une approche objective et une approche fondée sur la subjectivité de l'auteur»; «il est situé à la fois à l'intérieur de l'œuvre et au cœur de l'expérience et de la culture du lecteur». Appartenant à l'imaginaire collectif, le thème constitue donc une sorte de "pont» entre l'œuvre et le lecteur. Mais à travers l'analyse des Malavoglia de Verga, Romano Luperini montre comment il s'articule avec la formalisation spécifiquement littéraire qui participe de sa resémantisation comme, le fait du reste, son appartenance à une époque historique.

La deuxième partie est consacrée à l'articulation entre le vécu et la narration chez des ethnologues qui ont mené des enquêtes de terrain et ont éprouvé le besoin d'écrire un livre littéraire à un moment donné de leur histoire. Vincent Debaene relève que dans les années 1930 et 1940, beaucoup d'entre eux ont doublement rendu compte d'une même expérience sous des jours différents. Le premier de leurs livres pourrait être considéré comme plus scientifique et le second plus littéraire (voir Leiris, Lévi-Strauss). Vincent Debaene s'interroge alors sur les raisons du "paradoxe d'un recours à la littérature au moment même où l'on prétend s'en débarrasser pour accéder 
à la science». En partant du principe que raconter est une nécessité chez l'homme, Alberto Sobrero s'interroge sur «le rapport entre la manière dont la littérature raconte le monde et la manière dont le fait l'anthropologie, entre le besoin pour l'une d'une certaine forme de vérité et le besoin pour l'autre d'une autre forme de vérité». Il relève en particulier combien l'écriture littéraire peut aider à exprimer la posture même de l'anthropologue à la fois à l'intérieur et à l'extérieur de l'histoire, sans que cela autorise à penser toutefois que les deux types de narration puissent être confondus, le romancier inventant des histoires et l'anthropologue tentant d'expliquer le monde. C'est à partir de ces prémisses qu'il aborde la tentation du littéraire chez Malinovski et l'expérience de l'«écriture du dedans» chez Leiris.

La troisième partie porte sur des études de cas. Michela Lo Feudo analyse l'évolution d'une théorisation de «l'autre de l'art» par Champfleury, l'un des premiers à s'être interrogé sur le statut de la culture populaire sous toutes ses formes, et ses rapports avec l'écriture narrative et fictionnelle. Jean-Marie Privat, nous l'avons vu, aborde le texte de Madame Bovary par le biais de la célèbre casquette de Charles, une sorte de synecdoque des tensions culturelles qui régissent toute l'œuvre. Silvia Disegni relit certains romans des Goncourt et le Journal pour trouver dans leur production la manière dont la superstition, la magie et les croyances, véritables lieux de tension textuels, arrivent à travailler leurs enquêtes et leurs œuvres. Gilles Bonnet examine le regard porté par le narrateur sur la mort et les morts dans L'Enterrement de François Bon, où se donne à lire la remise en cause d'une anthropologie désuète à la faveur d'une autre idée de l'anthropologie qui joue un rôle majeur dans ce roman structuré à partir de l'opposition entre ces deux pôles.

Dans la dernière partie, consacrée à l'étude d'un thème et d'un mythe, Carlo Donà, d'abord, analyse, sur une longue durée et dans un texte très documenté, les transformations d'un thème qui connut une grande fortune pendant des siècles, celui de la puella venenata, la jeune fille empoisonnée et assassine, constamment resémantisé selon les époques et le contexte où il apparait. On le trouve en Orient puis en Occident, de l'Antiquité jusqu'à nos jours et dans des typologies de textes très variées, dont certaines sont de nature fictionnelle et d'autres scientifiques. Carlo Donà s'interroge alors sur les raisons de sa persistance. Enfin, dans une étude sur la nouvelle «Le Juif errant» d'Elie Wiesel, c'est la transformation du mythe homonyme qu'interroge Marcello Massenzio pour en montrer le renversement sémantique au moment de son appropriation par la culture juive. Wiesel relit le mythe à la lumière de son actualité, la Shoah. Massenzio s'interroge alors sur le rapport complexe qui s'instaure entre les deux temporalités, celle du mythe et celle du présent dont l'articulation permet de donner un sens nouveau au Juif errant, 
devenu «mémoire vivante» de la destruction du temple, capable d'insuffler aux rescapés l'énergie morale» servant à surmonter la catastrophe.

La diversité de ces approches témoigne de ce que peuvent gagner littérature et anthropologie de leur rencontre. Paradoxalement, tout en ouvrant le texte littéraire à l'interdisciplinarité, tout en explorant et en interrogeant ce dont il se nourrit, ou ce vers quoi il tend, certaines d'entre elles s'efforcent de ne pas remettre en cause la spécificité de celui-ci et se penchent sur la manière dont cette spécificité se construit dans le dialogue du premier avec l'autre de la littérature, ou dans le heurt avec cet autre, parfois dans une méfiance exhibée ou latente, parfois dans le dépassement de celle-ci; tantôt dans l'exaltation des tensions et des différences qui les opposent, tantôt dans l'adaptation et l'appropriation de la différence dans le texte au nom de la revendication d'une forte instance auctoriale. De même en va-t-il du rapport que les anthropologues entretiennent avec le fait littéraire lorsqu'ils y lisent une manière de renforcer leur propre questionnement ou confirmer leurs propres résultats ou, au contraire de les ouvrir à d'autres champs ou lieux d'enquête, d'autres modalités ou expressions du "discours sur l'homme». Dans un sens comme dans l'autre, c'est toujours l'idée de leur frontière qui est interrogée et constamment réinterrogée, dans un renvoi d'images de l'une à l'autre qui fait parfois vaciller l'idée même des limites qui les séparent.

\section{Remarque terminologique}

Le lecteur pourra être surpris de cette sorte d'indécision qui traverse l'ensemble des contributions qui suivent, où se côtoient les termes «ethnographie», «ethnologie» et «anthropologie», dont les relations historiques et la distinction ont fait l'objet de nombreux débats. Au cours de définitions successives, Lévi-Strauss a justifié l'usage des trois notions comme autant d'étapes d'un même parcours : la première, l'enquête de terrain empirique; la deuxième, l'étude comparée des documents recueillis et leur systématisation; la troisième enfin, plus théorique et intégrant les deux premières, la constitution d'une connaissance générale de l'homme, qui permet le dialogue avec d'autres sciences humaines aspirant à un même type de connaissance. Sans entrer dans le détail, et quitte à simplifier les choses, je me contenterai de rappeler qu'une telle indétermination, si répandue aujourd'hui, tient au fait assez communément admis qu'à l'intérieur de la même démarche, les différentes «étapes» ne sont pas à considérer en diachronie mais en synchronie, chacune présupposant l'autre - ou les autres - au moment même où elle prend forme. 